\title{
ON A CERTAIN NON-LINEAR ONE-PARAMETER SYSTEM OF HYPERSURFACES OF ORDER $n$ IN $r$-SPACE
}

\author{
BY B. C. WONG
}

Consider a linear $\infty^{\rho_{\text {-system, }} \text {, where }}$

$$
\rho \leqq \frac{(n+r) !}{n ! r !}-1
$$

of hypersurfaces of order $n$, which may have $\sigma$ base points, in an $r$-space, $S_{r}$. Denote this system by $|W|$.

Now let $\nu_{1}+\nu_{2}+\cdots+\nu_{t}$ projectively related curves

$$
C_{11}, C_{12}, \cdots, C_{1 \nu_{1}}, C_{21}, C_{22}, \cdots, C_{2 \nu_{2}}, \cdots, C_{t \nu_{t}}
$$

of orders

$$
n_{11}, n_{12}, \cdots, n_{1 \nu_{1}}, n_{21}, n_{22}, \cdots, n_{2 \nu_{2}}, \cdots, n_{t v_{t}},
$$

respectively, and all of genus $p$, be given in the same $r$-space $S_{r}$. To a point on any one of the curves corresponds a definite point on each of the other curves. We assume that none of the given curves passes through any of the $\sigma$ base points of $|W|$ and that none of the intersections, if there be any, of any two of the curves is a self-corresponding point. Let $P_{11}, P_{12}, \cdots, P_{t v_{t}}$ be a set of corresponding points, the point $P_{i j_{i}}$ being on the curve $C_{i j_{i}},\left(i=1,2, \cdots, t ; j_{i}=1,2, \cdots, \nu_{i}\right)$. If

$$
\nu_{1}+2 \nu_{2}+\cdots+t \nu_{t}=\rho \leqq \frac{(n+r) !}{n ! r !}-1,
$$

there is one and only one hypersurface of the system $|W|$ such that $1,2, \cdots, t$ of the points of its intersection with each of the $\nu_{1}$ curves $C_{1 j_{1}}, \nu_{2}$ curves $C_{2 j_{2}}, \cdots, \nu_{t}$ curves $C_{t j_{t}}$, will coincide with $P_{1 j_{1}}, P_{2 j_{2}}, \ldots, P_{t j_{t}}$, respectively. Denote such a hypersurface by $V_{r-1}^{n}$. As the corresponding points describe their respective curves, $V_{r-1}^{n}$ describes a non-linear one-parameter system, $\{V\}$, of hypersurfaces of order $n$ in $S_{r}$. In this paper we propose to determine the number, $N_{0}$, the order of the system, of the hypersurfaces of the system passing through a given 
point and also the number, $N_{k}$, of those tangent to a given $k$-space for $k=1,2, \cdots, r$. The symbol $N_{r}$ means the number of the hypersurfaces that have each a node.

In the following, we shall give two determinations of the number $N_{0}$ : the one by the use of the theory of correspondence and the other by the aid of the following known proposition.*

(A) Let there be given $q$ varieties $V_{x_{1}}^{m_{1}}, V_{x_{2}}^{m_{2}}, \cdots, V_{x_{q}}^{m_{q}}$ of orders $m_{1}, m_{2}, \cdots, m_{q}$, respectively, such that $V_{x_{i}}^{m_{i}}$ is the locus of $\infty^{1}\left(x_{i}-1\right)$-spaces. If there exists a one-to-one correspondence between the elements of these varieties, then the locus of the $\infty^{1}\left(x_{1}+x_{2}+\cdots+x_{q}-1\right)$-spaces determined by corresponding elements is a $V_{x_{1}+x_{2}+\cdots+x_{q}}$ of order $m_{1}+m_{2}+\cdots+m_{q}$.

We now determine $N_{0}$ by the theory of correspondence. We commence with the case $\nu_{1}=\rho=2, \nu_{2}=\nu_{3}=\cdots=\nu_{t}=0$. The system $\{V\}$ now consists of those hypersurfaces of the net $|W|$ which pass through pairs of corresponding points on the two given curves $C_{11}, C_{12}$. The desired number is the number of hypersurfaces of $\{V\}$ passing through a given point, say $A$. Let us make a hypersurface $W_{r-1}^{n}$ of $|W|$ pass through $A$ and a point $P_{11}$ of $C_{11}$. This $W_{r-1}^{n}$ meets $C_{12}$ in $n n_{12}$ points $Q_{12}, Q_{12}{ }^{\prime}, \cdots$. If one of these points happens to coincide with the point $P_{12}$ corresponding to $P_{11}$ on $C_{11}$, then $W_{r-1}^{n}$ is a $V_{r-1}^{n}$ of $\{V\}$. In general, this does not happen. Now pass another hypersurface $W_{r-1}^{\prime n}$ of $|W|$ through $A$ and one of the points $Q_{12}, Q_{12}{ }^{\prime}, \ldots$, say $Q_{12}$. This $W_{r-1}^{\prime n}$ meets $C_{11}$ in $n n_{11}$ points $P_{11}, P_{11}{ }^{\prime}, \cdots$, to which correspond $n n_{11}$ points $P_{12}, P_{12}{ }^{\prime}, \cdots$, on $C_{12}$. We see that we have thus established a correspondence on the curve $C_{12}$ such that to each of the points $Q_{12}, Q_{12}{ }^{\prime}, \ldots$ correspond $n n_{11}$ points $P_{12}$, $P_{12}{ }^{\prime}, \cdots$ and to each of the latter correspond $n n_{12}$ points of the former. If a united point occurs, then the two hypersurfaces $W_{r-1}^{n}, W_{r-1}^{\prime n}$ become coincident with a $V_{r-1}^{n}$ of $|V|$. The correspondence being obviously of valence zero, the number of united points, and therefore the order of $\{V\}$, is $n\left(n_{11}+n_{12}\right)$.

Suppose now $\nu_{1}=\rho=3, \nu_{2}=\nu_{3}=\cdots=\nu_{t}=0$. Choose a $W_{r-1}^{n}$ of the web $|W|$ that passes through a given point $A$ and a pair

* A full discussion of this proposition is found in Edge, On the quartic developable, Proceedings of the London Mathematical Society, (2), vol. 33, pp. 52-65. The statement above is quoted verbatim from B. C. Wong, On the number of stationary tangent $S_{t-1}$ 's to $a V_{k}^{n}$ in an $S_{t k+k-1}$, this Bulletin, vol. 39 (1933), pp. 608-610. 
of corresponding points $P_{11}, P_{12}$ on $C_{11}, C_{12}$. This $W_{r-1}^{n}$ meets the third given curve $C_{13}$ in $n n_{13}$ points $Q_{13}, Q_{13}{ }^{\prime}, \ldots$, none of which, in general, coincides with the point $P_{13}$ corresponding to $P_{11}$ and $P_{12}$. Through each of these points $Q_{13}, Q_{13}{ }^{\prime}, \cdots$ there are, according to the result just found, $n\left(n_{11}+n_{12}\right)$ hypersurfaces each containing $A$ and a pair of points $P_{11}, P_{12}$. Then on $C_{13}$ there are $n\left(n_{11}+n_{12}\right)$ points $P_{13}, P_{13}{ }^{\prime}, \cdots$ corresponding to as many pairs on $C_{11}, C_{12}$. Now we have on $C_{13}$ an $\left(n_{11} n+n_{12} n, n_{13} n\right)$-correspondence of valence zero between the points $P_{13}, P_{13}{ }^{\prime}, \ldots$ and the points $Q_{13}, Q_{13}{ }^{\prime}, \cdots$. The number of united points, and therefore the number of the hypersurfaces of $|W|$ passing through $A$ and a set of corresponding points on $C_{11}, C_{12}, C_{13}$, is $n\left(n_{11}+n_{12}+n_{13}\right)$.

If we continue reasoning in this manner, we shall find that, for the case $\nu_{1}=\rho, \nu_{2}=\nu_{3}=\ldots=\nu_{t}=0$, the order of $\{V\}$ is $n\left(n_{11}+n_{12}+\cdots+n_{1 \nu_{1}}\right)$ or $n \sum_{j_{1}=1}^{v_{1}} n_{1 j_{1}}$.

Now suppose $\nu_{1}=\rho-2, \nu_{2}=1$. Then the system $\{V\}$ consists of all those hypersurfaces of the $\infty^{\rho_{\text {-system }}}|W|$ which contain a set of corresponding points $P_{1 j_{1}},\left(j_{1}=1,2, \cdots, \nu_{1}\right)$, on the $\nu_{1}$ curves $C_{1 j_{1}}$ and have a contact with $C_{21}$ at the point $P_{21}$ corresponding to $P_{1_{11}}$. Select a $W_{r-1}^{n}$ of $|W|$ passing through a given point $A$ and a fixed set of points $P_{1 j_{1}}$ and having a point of contact with $C_{21}$. Since a pencil of hypersurfaces of order $n$ contains $2\left(n_{21} n-1+p\right)$ members tangent to a given curve of order $n_{21}$ and genus $p$, there are $2\left(n_{21} n-1+p\right)$ such hypersurfaces and hence there are as many points of contact $T_{21}, T_{21}{ }^{\prime}, \cdots$ on $C_{21}$. None of these, in general, coincides with $P_{21}$. Now pass a hypersurface $W_{r-1}^{\prime n}$ of $|W|$ through the points $P_{1 j_{1}}$ on the curves $C_{1 j_{1}}$ tangent to $C_{21}$ at one of the points $T_{i 1}, T_{21}{ }^{\prime}, \cdots$, say $T_{21}$. There are, according to the result of the preceding paragraph, $n \sum_{j_{1}=1}^{\nu_{1}} n_{1 j_{1}}$ such hypersurfaces giving rise to as many sets of corresponding points $P_{1_{1}}, P_{1_{j_{1}}}^{\prime}, \ldots$, to which correspond as many points $P_{21}, P_{21}^{\prime}, \cdots$, on $C_{21}$. Thus, we have established an $\left[n \sum_{j_{1}=1}^{\nu_{1}} n_{1 j_{1}}, 2\left(n_{21} n-1+p\right)\right]$-correspondence also of valence zero between the points $P_{21}, P_{21}, \ldots$, and the points $T_{21}, T_{21}{ }^{\prime}, \cdots$, on $C_{21}$. The number of united points in this correspondence which gives the order of $\{V\}$ is therefore $n \sum_{j_{1}-1}^{\nu_{1}} n_{1 j_{1}}+2\left(n_{21} n-1+p\right)$.

These particular cases are sufficient to indicate the method used. Reasoning in exactly the same manner for all the differ- 
ent values of the $\nu$ 's satisfying (I), we find the general result

$$
\begin{aligned}
& \text { (1) } N_{0}=\sum_{j_{1}=1}^{\nu_{1}} n_{1 j_{1}} n+2 \sum_{j_{2}=1}^{\nu_{2}}\left(n_{2 j_{2}} n-1+p\right) \\
& +3 \sum_{j_{3}=1}^{\nu_{3}}\left(n_{3 j_{3}} n-2+2 p\right)+\cdots+t \sum_{j_{t}=1}^{\nu_{t}}\left[n_{t j_{t}} n+(t-1)(p-1)\right],
\end{aligned}
$$

or

$$
N_{0}=\sum_{i=1}^{t} i \sum_{j_{i}=1}^{\nu_{i}}\left[n_{i j_{i}} n+(i-1)(p-1)\right] \text {, }
$$

where $i\left[n_{i j_{i}} n+(i-1)(p+1)\right]$ is the number of hypersurfaces of order $n$ of an $\infty^{i-1}$-system of hypersurfaces such that $i$ of the points of intersection of each of them with a given curve of order $n_{i j_{i}}$ are coincident.

Now we determine $N_{0}$ by the aid of (A). Let the hypersurfaces of $|W|$, which may have $\sigma$ base points, represent upon $S_{r}$ an $r$-dimensional variety $\Phi_{r}{ }^{n^{r}-\sigma}$ of order $n^{r}-\sigma$ in a $\rho$-space $S_{\rho}$. The $\nu_{1}+\nu_{2}+\cdots+\nu_{t}$ given curves, none of which is supposed to pass through any of the $\sigma$ base points, are the images of curves $\Gamma_{i j_{i}}$ of order $n_{i j_{i}} n$ on $\Phi_{r}{ }^{n^{r}-\sigma}$ whose points are also in a one-to-one correspondence. Let $R_{11}, R_{12}, \cdots$ be a set of corresponding points, the point $R_{i j_{i}}$ being on the curve $\Gamma_{i j_{i}}$. Corresponding to a hypersurface $V_{r-1}^{n}$ of the system $\{V\}$ is a section $\Theta_{r-1}^{n^{r}-\sigma}$ of $\Phi_{r}^{n r-\sigma}$ by a $(\rho-1)$-space which contains a set of points $R_{1 j_{1}}$ on the curves $\Gamma_{1 j_{1}}$, a set of tangent lines at the points $R_{2 j_{2}}$ on the curves $\Gamma_{2 j_{2}}$, a set of osculating planes at the points $R_{3 j_{3}}$ on the curves $\Gamma_{3 j_{3}}, \cdots$. The $\infty^{1}(\rho-1)$-spaces of the nature just described form an $\infty^{1}$-system to which corresponds our system $\{V\}$ of hypersurfaces. By applying (A) we find that the order of the system of $(\rho-1)$-spaces is, since the $i$-dimensional developable of the curve $\Gamma_{i j_{i}}$ is of order $i\left[n_{i j_{i}} n+(i-1)(p-1)\right]$, the same as (1). Now through a given point $A^{\prime}$ which may be, without loss of generality, placed upon $\Phi_{r}{ }^{n^{r}-\sigma}$, pass the same number of $(\rho-1)$-spaces of the system and each such $(\rho-1)$ space intersects $\Phi_{r}^{n^{r}-\sigma}$ in a $\Theta_{r-1}^{n^{r}-\sigma}$ passing through $A^{\prime}$ to which corresponds a $V_{r-1}^{n}$ of $\{V\}$ passing through a given point $A$, the image of $A^{\prime}$. Thus, the determination is complete.

Hitherto we have assumed that none of the given curves 
passes through any of the base points of $|W|$ and that none of the intersections, if there be any, of any two of the curves is a self-corresponding point. If, however, a curve $C_{i i_{i}}$ passes through one of the base points, we must deduct $i$, and if any two whatever of the curves intersect in a self-corresponding point, we must deduct unity from the general value of $N_{0}$ which we have just derived.

As an example consider a linear $\infty^{6}$-system $|K|$ of quartic curves in a plane $\phi$ with 8 base points. Let three projectively related cubic curves $\gamma^{3}, \gamma^{\prime 3}, \gamma^{\prime \prime 3}$ of genus unity be given in the plane, none of the intersections of the curves being a self-corresponding point. Select a quartic of $|K|$ such that one of its intersections with $\gamma^{3}$ coincides at $P$, two of its intersections with $\gamma^{\prime 3}$ coincide at $P^{\prime}$, and three of its intersections with $\gamma^{\prime \prime 3}$ coincide at $P^{\prime \prime}$, where $P, P^{\prime}, P^{\prime \prime}$ are a set of corresponding points. There are $\infty^{1}$ such quartic curves forming a non-linear pencil, $\{C\}$. Now the quartics of $|K|$ represent upon $\phi$ a surface $\Phi^{8}$ of order 8 in $S_{6}$ upon which lie three projectively related curves $\Gamma^{12}, \Gamma^{\prime 12}, \Gamma^{\prime \prime 12}$ all of order 12 and genus 1 , of which $\gamma^{3}, \gamma^{\prime 3}, \gamma^{\prime \prime 3}$ are the images in $\phi$. The locus of tangent lines of $\Gamma^{\prime 12}$ is of order 24 and the locus of osculating planes of $\Gamma^{\prime \prime 12}$ is of order 36 . Let $R, R^{\prime}, R^{\prime \prime}$ be a set of corresponding points on the curves. Then we say that the tangent $t^{\prime}$ to $\Gamma^{\prime 12}$ at $R^{\prime}$ and the osculating plane $\pi^{\prime \prime}$ to $\Gamma^{\prime \prime 12}$ at $R^{\prime \prime}$ correspond to the point $R$ on $\Gamma^{12}$. The 5 -spaces determined by $R, t^{\prime}, \pi^{\prime \prime}$ will describe an $\infty^{1}$-system of 5 -spaces such that $N_{0}=72$ of them pass through a given point $A^{\prime}$ which may be placed on $\Phi^{8}$. Therefore the system $\{C\}$ of quartic curves contains 72 members passing through a given point $A$ of $\phi$.

Suppose the curve $\gamma^{3}$ passes through a base point of $|K|$. Then the corresponding curve $\Gamma^{12}$ on $\Phi^{8}$ is composed of a $\Gamma^{11}$ and a line. Discarding the line or deducting unity, we have $N_{0}=71$. If $\gamma^{\prime 3}$ alone contains a base point, the corresponding curve $\Gamma^{\prime 12}$ degenerates into a line, to be disregarded, and a $\Gamma^{\prime 11}$ whose developable surface is of order 22 . Therefore, we deduct 2 and now $N_{0}=70$. Finally, let $\gamma^{\prime \prime 3}$ alone go through a base point. The curve $\Gamma^{\prime \prime 12}$ on $\Phi^{8}$ is made up of a line, also to be disregarded, and a $\Gamma^{\prime \prime 11}$ the locus of whose osculating planes is of order 33. Deducting 3, we now have $N_{0}=69$.

Now let one of the intersections of $\gamma^{3}, \gamma^{\prime 3}$ be a self-correspond- 
ing point. Then $\Gamma^{12}, \Gamma^{\prime 2}$ also have a self-corresponding point $R \equiv R^{\prime}$ to which corresponds the point $R^{\prime \prime}$ on $\Gamma^{\prime \prime 12}$. There is a linear pencil of 5 -spaces passing through the tangent line $t^{\prime}$ at the self-corresponding point $R \equiv R^{\prime}$ and containing the osculating plane of $\Gamma^{\prime \prime 12}$ at $R^{\prime \prime}$. Disregarding this pencil, we deduct 1 and the result is $N_{0}=71$. If $\gamma^{\prime 3}, \gamma^{\prime \prime 3}$ have a self-corresponding point in common, then one of the intersections of $\Gamma^{\prime 12}, \Gamma^{\prime \prime 12}$ is a self-corresponding point $R^{\prime} \equiv R^{\prime \prime}$ to which corresponds a point $R$ of $\Gamma^{12}$. There is a linear pencil of 5 -spaces determined by $R$ and the tangent line to $\Gamma^{\prime 12}$ at $R^{\prime}$ and the osculating plane of $\Gamma^{\prime \prime 12}$ at $R^{\prime \prime} \equiv R^{\prime}$. Deducting 1 , we have $N_{0}=71$.

We shall next proceed to determine $N_{k}$, the number of the hypersurfaces of $\{V\}$ tangent to a given $k$-space in $S_{r}$. We find it convenient to use the following method. We set up a one-toone correspondence between the points of a $\rho$-space $S_{\rho}$ and the hypersurfaces of the $\infty^{\rho}$-system $|W|$. Corresponding to the $\infty^{p-1}$ hypersurfaces of $|W|$ that pass through a given point $A$ are the $\infty^{\rho-1}$ points of a $(\rho-1)$-space $S_{\rho-1}$ of $S_{\rho}$, and corresponding to the hypersurfaces of $\{V\}$ are the points of a curve $\Delta$. Since there are given by (1), as we have seen, $N_{0}$ hypersurfaces of $|W|$ passing through $A$ and belonging to $\{V\}$, there must be $N_{0}$ points of $S_{\rho}$ common to $S_{\rho-1}$ and $\Delta$. Hence $\Delta$ is of order $N_{0}$.

Let a $k$-space $S_{k}$ be given in $S_{r}$. Contact being one condition, there are $\infty^{\rho-1}$ hypersurfaces of $|W|$ tangent to $S_{k}$, and to these contact hypersurfaces correspond $\infty^{\rho-1}$ points of a locus $\Sigma_{\rho-1}^{M}$ in $S_{\rho}$. By the methods of analytic geometry we find without difficulty that the order $M$ of $\Sigma_{\rho-1}^{M}$ is $M=(k+1)(n-1)^{k}$. All those hypersurfaces of $|W|$ belonging to $\{V\}$ and tangent to $S_{k}$ are given by all those points of $S_{\rho}$ common to $\Delta^{N_{0}}$ and $\Sigma_{\rho-1}^{M}$. Therefore, the number of hypersurfaces of $\{V\}$ tangent to $S_{k}$ is the number of the points in which $\Delta^{N_{0}}$ intersects $\Sigma_{\rho-1}^{M}$ and is therefore equal to $N_{k}=M N_{0}=(k+1)(n-1)^{k} N_{0}$.

For $k=1,2$, then, $N_{1}=2(n-1) N_{0}$ and $N_{2}=3(n-1)^{2} N_{0}$ are, respectively, the number of hypersurfaces of $\{V\}$ tangent to a given line and the number of those tangent to a given plane. If $k=r$, we have $N_{r}=(r+1)(n-1)^{r} N_{0}$ members of the system that have each a node.

The University of California 